\title{
Quantitative Mapping of Unmodulated Temperature Fields with Nanometer Resolution
}

\author{
Amin Reihani ${ }^{1}$, Yuxuan Luan ${ }^{l}$, Shen Yan ${ }^{l}$, Ju Won Lim ${ }^{2}$, Edgar Meyhofer ${ }^{1}$, Pramod Reddy ${ }^{1,2, *}$ \\ ${ }^{1}$ Department of Mechanical Engineering, University of Michigan, Ann Arbor, MI 48109 \\ ${ }^{2}$ Department of Materials Science and Engineering, University of Michigan, Ann Arbor, MI 48109 \\ *meyhofer@umich.edu,pramodr@umich.edu
}

\section{S1. Scanning Thermal Probe Fabrication}

The fabrication steps of the scanning thermal probes (STPs) employed in this study are illustrated in Figure 1. Briefly, a T-beam was first defined on a bare Si wafer by deep reactive ion etching (DRIE) of Si. This was followed by wet oxidation of $\mathrm{Si}$ and low-pressure chemical vapor deposition (LPCVD) of $600 \mathrm{~nm}$ silicon nitride on the etched Si substrate which formed the Tbeam. The purpose of the T-beams was to improve the stiffness of the probe cantilever which enabled a stable thermal contact with the sample during thermal scanning, ${ }^{1}$ while simultaneously minimizing the thermal conductance of the probe. Next, the back side of the wafer was patterned by reactive ion etching (RIE). Subsequently, we deposited a $3 \mu \mathrm{m}$ thick low temperature oxide (LTO) with Cr caps defined on the front side to prepare for formation of conical tips in the next step. Then, the LTO was etched by buffered HF (BHF), leaving a probe tip with a height of $\sim 3 \mu \mathrm{m}$ under the region where the $\mathrm{Cr}$ cap was previously located. Afterwards, the tip was coated by a 150 $\mathrm{nm}$ Cr layer. Next, we deposited and patterned a $50 \mathrm{~nm}$ thick serpentine Pt line to form a Pt heaterthermometer for resistive heating and thermometry on the probe. Later, we deposited and patterned the $\mathrm{Au}$ reflector and electrodes. Next, the front side of the wafer was covered by a $50 \mathrm{~nm}$ thick 
plasma-enhanced chemical vapor deposition (PECVD) nitride layer to protect it by reducing the etching rate during the final etch step. Finally, the probe was released and a suspended thermal reservoir was formed on the probe body by $\mathrm{KOH}$ etching of $\mathrm{Si}$ (step 9 of Figure S1).
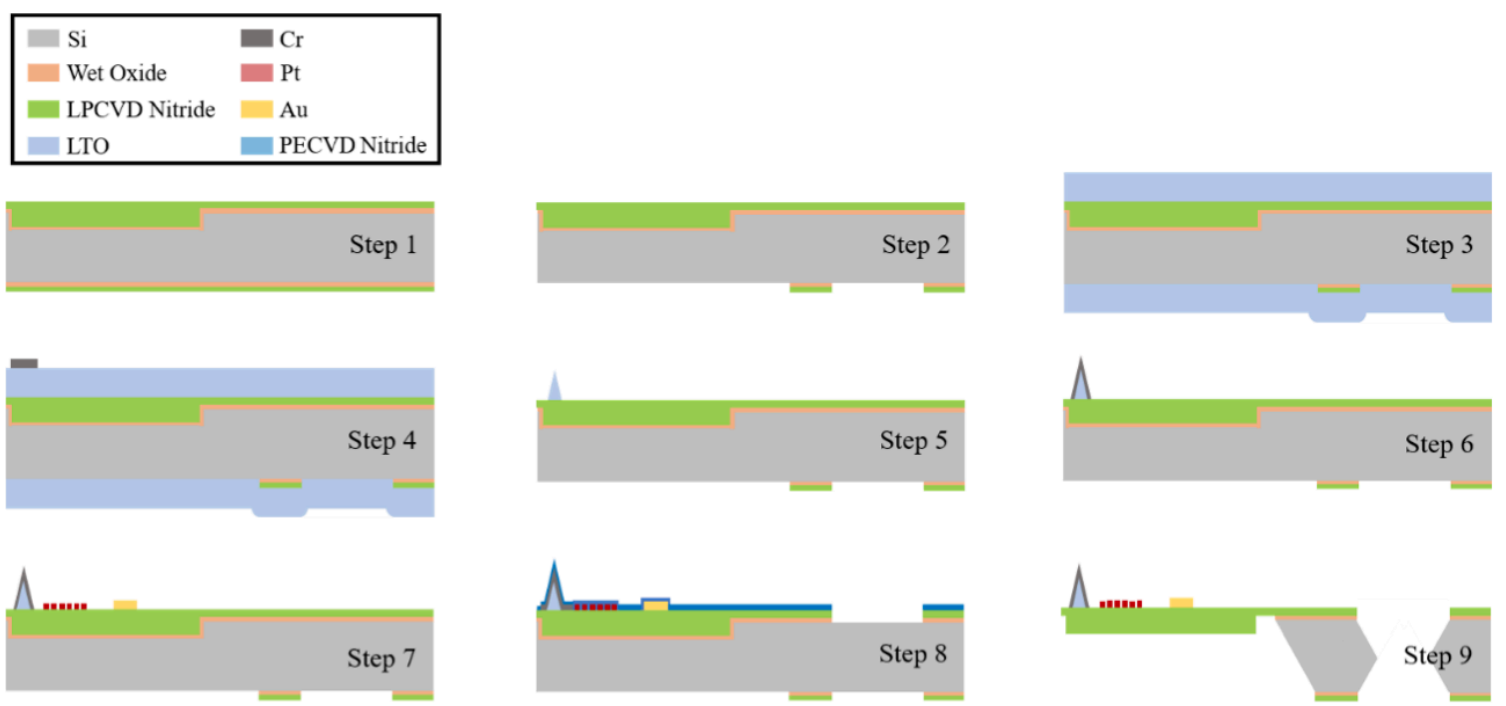

Figure S1 Process steps for fabrication STPs with integrated platinum resistive heater/thermometer and thermal reservoir temperature sensing capability

\section{S2. Mechanical stiffness and thermal conductance of scanning thermal probe}

The mechanical stiffness of the scanning thermal probe was estimated by performing finite element simulations in $\mathrm{COMSOL}^{\mathrm{TM}}$. The probe geometry including various layers of $\mathrm{Au}, \mathrm{Pt}$ and $\mathrm{Cr}$ were incorporated into the model. The probe is $215 \mu \mathrm{m}$ long and is formed from $600 \mathrm{~nm}$ thick $\mathrm{SiN}_{\mathrm{x}}$ films with thin films of Pt $(50 \mathrm{~nm}), \mathrm{Au}(100 \mathrm{~nm})$ and $\mathrm{Cr}(150 \mathrm{~nm})$ patterned on it. In order to estimate the mechanical stiffness we imposed a fixed boundary condition at the base of the probe and a force of $F=1 \mu \mathrm{N}$ was applied to the probe tip resulting in a deflection of $\delta=0.13 \mu \mathrm{m}$. From these data (see Figure 2a) we estimate the probe stiffness to be $\sim 7.69 \mathrm{~N} / \mathrm{m}$. 
The thermal resistance of the probe was also studied using the aforementioned model by considering a thermal conductivity of $8.5 \mathrm{~W} / \mathrm{m} \cdot \mathrm{K}$ and a thermal diffusivity of $1.5 \times 10^{-6} \mathrm{~m}^{2} / \mathrm{s}$ for $\mathrm{SiN}_{\mathrm{x}}$ thin films. ${ }^{2} \mathrm{~A}$ constant temperature $\left(20^{\circ} \mathrm{C}\right)$ boundary condition was chosen for the probe base, while a distributed heat flux was applied to the Pt heater on the probe (as shown by the inset in Figure S2b), which generated a temperature gradient along the probe body. The temperature rise of the tip was monitored under various input heat fluxes and the thermal resistance of the probe was extracted as the slope of linear fit to the data shown in Figure $\mathrm{S} 2$ (b) to be $R_{\mathrm{P}}=0.51 \times 10^{6} \mathrm{~K} / \mathrm{W}$ around $300 \mathrm{~K}$ which is in good agreement with the experimental measurements shown in Figure $2 \mathrm{~b}$ of the manuscript.

a)

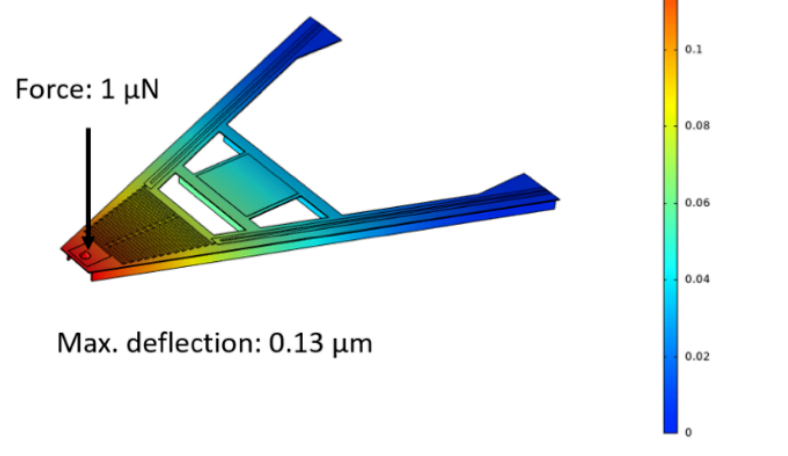

b) 3

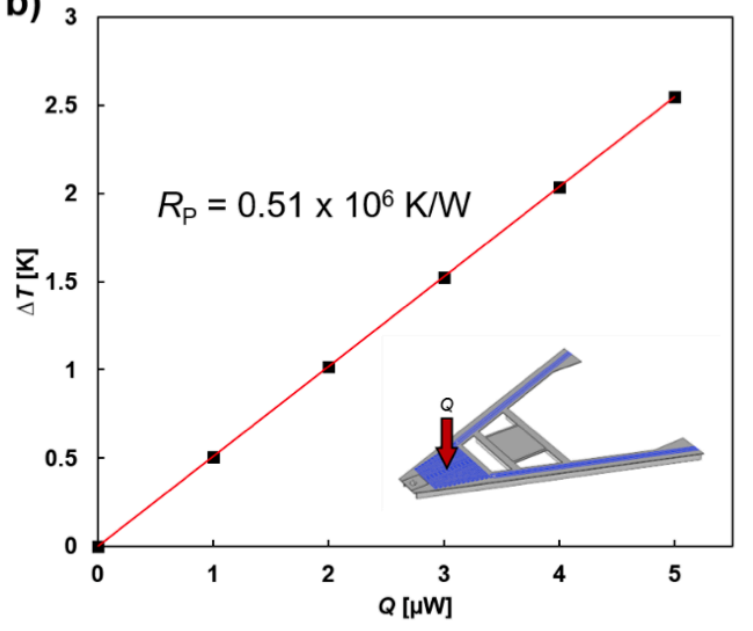

Figure S2 (a) Finite element simulation of STP deflection under an applied load. (b) Temperature rise of the probe as a function of heat input to the tip. The slope of the linear fit shows the probe thermal resistance $R_{P}$.

\section{S3. Sample temperature stabilization}

The sample temperature was measured by mounting a glass encapsulated thermistor (TDK Electronics B57550G1103F000) on the surface of the sample chip, $\sim 3 \mathrm{~mm}$ away from the center of the sample (where the Pt line was located), using silver paste to make a strong thermal contact. 
The temperature of the sample was measured using a Wheatstone bridge driven by a sine wave with a peak amplitude of $200 \mathrm{mV}$ at a frequency of $320 \mathrm{~Hz}$ and a lock-in technique. The temperature was controlled using a PID controller, with gains tuned using the Ziegler-Nichols method, ${ }^{3}$ at a bandwidth of $4 \mathrm{~Hz}$ to a stability level of $\sim 100 \mu \mathrm{K}$ as shown in the time-series measurement of Figure S3. To heat the sample to $26^{\circ} \mathrm{C}$, a filament heater integrated into the sample holder of the atomic force microscope (UHV 750, Beetle STM/AFM, RHK Technology) was employed which radiatively heated the sample from back side.

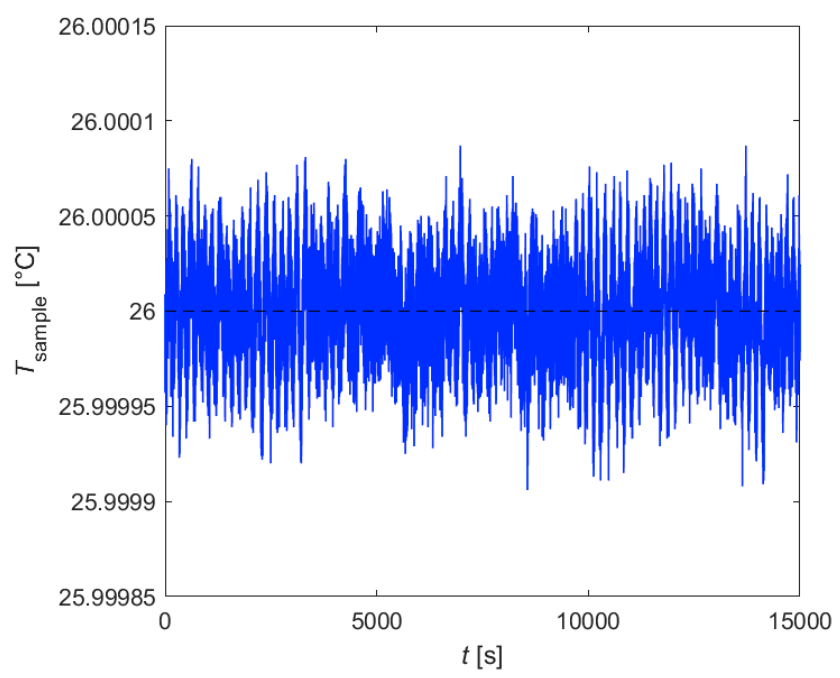

Figure S3 The measured bulk temperature of the feedback-stabilized sample as a function of time.

\section{S4. Self-heated Pt line sample temperature rise measurement}

In order to verify that our SThM measurements accurately quantify the sample temperature, we need an independent measure of temperature rise of the heated Pt line sample when an electrical current is supplied to the Pt line. To this end, we used the temperature dependent resistance of the Pt line to independently measure its average temperature rise. In this measurement, the sample was placed in a half Wheatstone bridge circuit (see Ref. ${ }^{4}$ for details) and an AC current at a frequency 
of $f=2 \mathrm{~Hz}$ at various amplitudes was sourced through the Pt line. Subsequently, the corresponding temperature modulations of the sample were measured using a $3 \omega$ method via the relation ${ }^{1}$ $\Delta T(2 f)=2(\mathrm{~d} T / \mathrm{d} R) V_{3 \mathrm{f}} / I_{0}=2 V_{3 \mathrm{f}} /\left(\alpha R I_{0}\right)$. In this relation $V_{3 \mathrm{f}}$ is the voltage output of the aforementioned circuit at a frequency $3 f, I_{0}$ is the amplitude of sinusoidal heating current, $\alpha$ and $R$ are the temperature coefficient of resistance and the resistance of the Pt line (measured using the 4-probe method) respectively. Figure S4 shows the results of this measurement which is the average temperature modulation amplitude of the heated Pt line as a function of the square of heating current amplitude $\left(I_{0}^{2}\right)$ sourced to the Pt line.

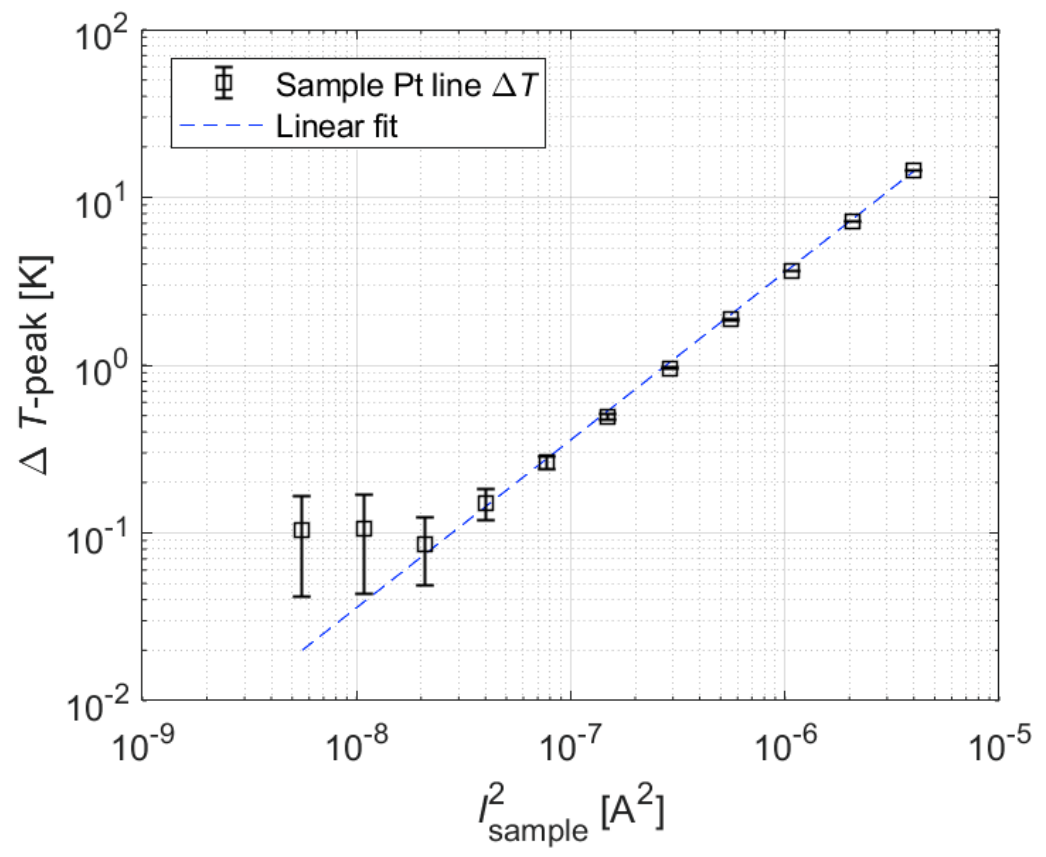

Figure S4 Temperature rise of the heated Pt line (250 nm wide, $50 \mathrm{~nm}$ thick, $18 \mu \mathrm{m}$ long) as a function of square of heating current amplitude.

\section{S5. Validity of lumped capacitance thermal model of the scanning thermal probe}

To check the validity of the lumped capacitance model (Figure 2a of manuscript) in describing the dynamic thermal behavior of the scanning thermal probe (STP) which is a continuum system, we 
performed a transient finite element heat conduction simulation in COMSOL for the exact geometry and the same materials from which our STP is made. Figure S5 shows the geometry and boundary conditions employed in this model. The temperature at one end of the cantilever is fixed at $300 \mathrm{~K}$, and a distributed heat input $Q$ is applied to the Pt serpentine and connecting lines (shown as blue regions in Figure S5). We modelled the effect of tip-sample contact by associating a thermal resistance $\left(R_{\mathrm{TS}}\right)$ with the contact to a sample at $300 \mathrm{~K}$.

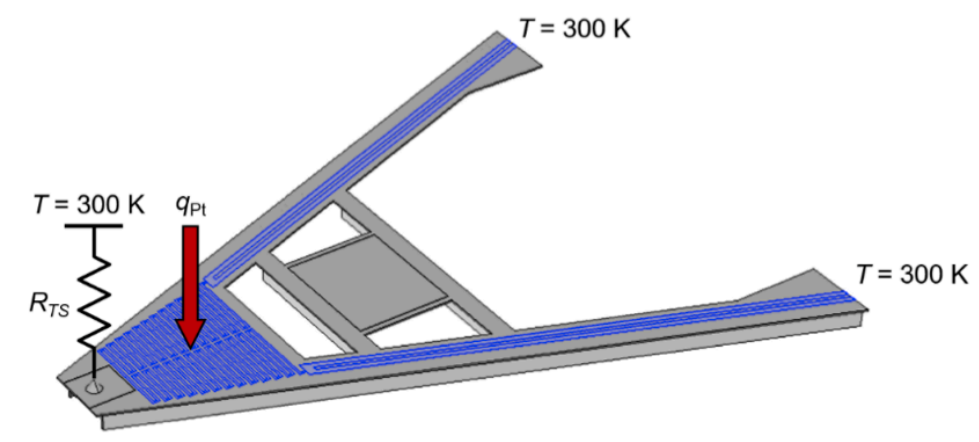

Figure S5 The geometry of the scanning thermal probe (STP) and the boundary conditions used in a finite element transient thermal model in COMSOL.

First, we performed a calculation for conditions where the probe is out of contact (i.e. $R_{\mathrm{TS}}$ $=\infty)$. We applied a step input in the distributed heating power $(Q)$ starting at $t=0$ and modeled the thermal response of the probe which is shown in Figure S6a (black squares) in terms of normalized temperature rise $\Delta T / \Delta T_{\max }$. We fit this continuum model data with a curve that has the functional form of a lumped capacitance model: $\Delta T=\Delta T_{\max }(1-\exp (-t / \tau))$, where $t$ is the time from the start of the step input and $\tau$ is thermal time constant. The best fit curve to the continuum model data is shown in Figure S6a (blue line) and corresponds to a thermal time constant $\tau=17.0$ ms. From the good agreement between the modelled data and the fit we can conclude that the lumped capacitance model works well to describe the dynamic thermal behavior of the scanning 
thermal probe. Further, we note that the modelled time constant $(17.0 \mathrm{~ms})$ and experimentally observed time constants $(17.9 \mathrm{~ms})$ are in good agreement with each other.

Next, we applied a tip-sample thermal resistance boundary condition of $R_{\mathrm{TS}}=4 \mathrm{MK} / \mathrm{W}$ (in the range of experimentally observed values) to model the thermal contact to a sample at $300 \mathrm{~K}$ and computed the temperature distribution on the probe with a heat input of $5 \mu \mathrm{W}$. Figure S6b shows the steady state temperature distribution on the STP, which indicates that most of the temperature drop occurs in the beams, and the platinum resistance thermometer (PRT) sensing element of the probe has a relatively uniform temperature. As can be seen in the inset of Figure S6b, no significant gradient $(<1.5 \%$ of the total thermal differential) is observed near the tip or in the region between the tip and the PRT.
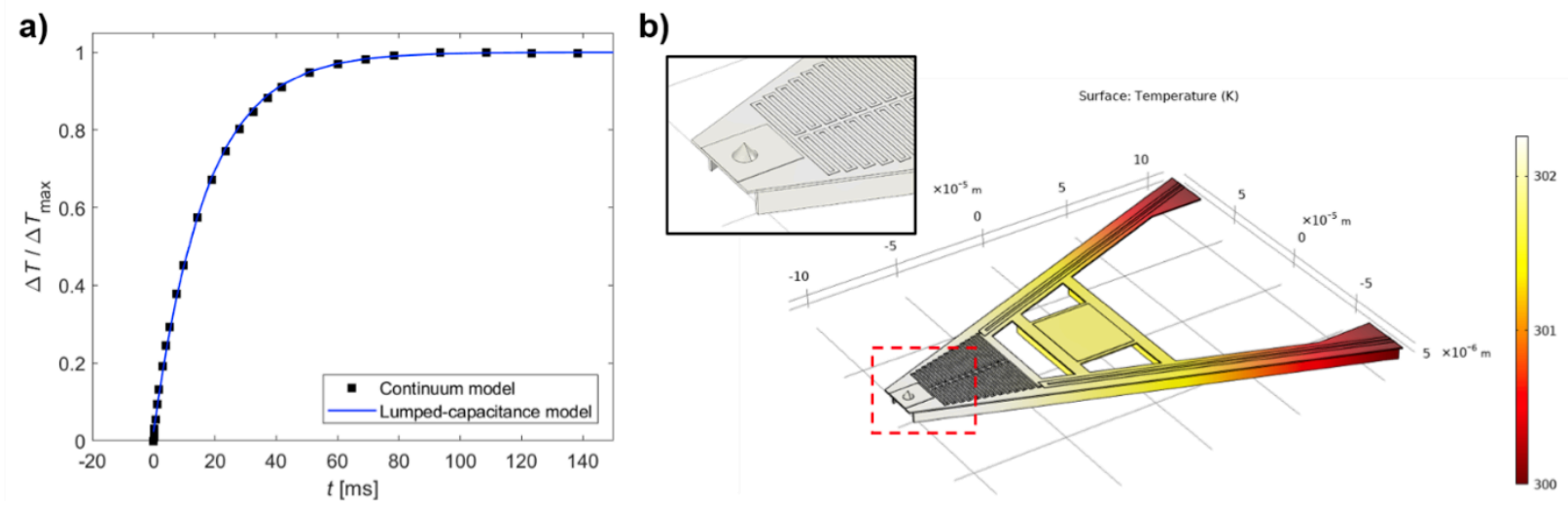

Figure S6 Simulated thermal response of the probe to a step input in heat flow to the Pt heater on the probe. (a) Shows the results of a continuum finite element model as well as a lumped capacitance model. (b) Steady-state temperature distribution in the probe as a result of a $5 \mu \mathrm{W}$ distributed heat input to the Pt heater and a tip-sample thermal contact resistance of $R_{T S}=4 \mathrm{MK} / \mathrm{W}$ to a sample at $300 \mathrm{~K}$. The temperature differential observed in the region shown in the inset is $<1.5 \%$ of the total temperature differential in the probe. 


\section{S6. Lumped capacitance thermal model of scanning thermal probe}

Based on the equivalent thermal circuit of Figure 2 (a) one can relate the probe temperature as sensed by the platinum resistance thermometer (PRT) close to the tip $\left(T_{\mathrm{P}}\right)$ to the input heat flux $\left(\dot{q}_{\mathrm{P}}\right.$ $(t))$ and the surface temperature $\left(T_{\mathrm{S}}\right)$ via: ${ }^{5}$

$$
\dot{q}_{\mathrm{P}}(t)=\frac{T_{\mathrm{P}}(t)-T_{\mathrm{R}}}{R_{P}}+\frac{T_{\mathrm{P}}(t)-T_{\mathrm{S}}}{R_{\mathrm{TS}}}+C_{P} \frac{d T_{\mathrm{P}}(t)}{d t}
$$

Here, $R_{\mathrm{P}}$ and $R_{\mathrm{TS}}$ are the probe-to-reservoir and probe tip-to-sample thermal resistances respectively, $C_{\mathrm{P}}$ is the lumped probe thermal capacitance, and $\dot{q}_{\mathrm{P}}$ is the heat flow deposited on the probe either by the integrated probe heater or the AFM laser. Note that in this analysis, the $R_{\mathrm{TS}}$ value is the sum of three thermal resistance components in series which are the PRT-tip thermal resistance (constant value), the tip-sample interface thermal resistance (which varies dynamically during the scan) and the spreading thermal resistance of the sample (which also varies dynamically during the scan) to a region of the sample whose temperature is not perturbed by probe contact. In our measurements, the tip-sample contact diameter is estimated to be $\sim 6 \mathrm{~nm}$ (see section $\mathrm{S} 8$ ), which results in a temperature decay depth of $\sim 3.5 \mathrm{~nm}$ into the sample (obtained using the analytic solution to heat conduction of a disk shaped heat source on a semi-infinite body $\left.{ }^{6}\right)$. Therefore, $R_{\mathrm{TS}}$ is the total thermal resistance from the probe PRT sensing element to a depth of few nanometers into the sample where the sample temperature is not perturbed. Similarly, the obtained sample temperature value in this analysis $\left(T_{\mathrm{S}}\right)$ is the unperturbed temperature of the sample corresponding to a depth of few nanometers into the sample.

For convenience, we define $R_{\text {tot }}$ as the total thermal resistance of the probe to the environment (i.e. thermal reservoir and sample): 
$\frac{1}{R_{\mathrm{tot}}}=\frac{1}{R_{\mathrm{P}}}+\frac{1}{R_{\mathrm{TS}}}$

Eq. S2

In our experiments, we source an $\mathrm{AC}$ current at frequency $f_{1}$ to the integrated probe heater which generates an AC heat flux via Joule heating at angular frequency $\omega=4 \pi f_{1}$. Therefore, the total probe heat flux $\left(\dot{q}_{\mathrm{P}}(t)\right)$ has a DC and AC component as shown below:

$$
\dot{q}_{\mathrm{P}}(t)=\dot{q}_{\mathrm{DC}}+\dot{q}_{\mathrm{AC}} \cos (\omega t)
$$

Based on Eq. S1, this heat input generates a DC and AC temperature change at the probe tip as shown in Eq. S4, where $\varphi$ is the phase shift of temperature response with respect to the heat input.

$$
T_{\mathrm{P}}(t)=T_{\mathrm{DC}}+T_{\mathrm{AC}} \cos (\omega t+\varphi)
$$

Now consider a calibration experiment, where the probe is retracted from the surface so that there is no thermal contact and $R_{\mathrm{TS}} \rightarrow \infty$ (i.e. $R_{\mathrm{TS}} \gg R_{\mathrm{C}}$ ). We label the $\mathrm{DC}$ and AC probe temperatures in out-of-contact (or open) condition as $T_{\mathrm{DC}-\mathrm{o}}$ and $T_{\mathrm{AC}-\mathrm{o}}$. Assuming small variation of probe temperature in out of contact condition compared to in contact condition, $\left|T_{\mathrm{DC}-\mathrm{o}}-\mathrm{T}_{\mathrm{DC}}\right|<5 \mathrm{~K}$, the variation in probe heater resistance would be $<1 \%$ and can safely be ignored. In addition, under the assumption of small probe temperature variations one can assume temperature independent probe thermal properties $\left(R_{\mathrm{P}}\right.$ and $\left.C_{\mathrm{P}}\right)$. Substituting Eq. S3 and Eq. S4 in Eq. S1, we obtain for the in contact case the following:

$$
\begin{aligned}
& T_{\mathrm{DC}}=R_{\mathrm{tot}}\left(\dot{q}_{\mathrm{DC}}+\frac{T_{\mathrm{R}}}{R_{\mathrm{P}}}+\frac{T_{\mathrm{S}}}{R_{\mathrm{TS}}}\right) \\
& T_{\mathrm{AC}}=\frac{R_{\mathrm{tot}} \dot{q}_{\mathrm{AC}}}{\sqrt{1+\left(R_{\mathrm{tot}} C_{\mathrm{P}} \omega\right)^{2}}} \\
& \varphi=-\tan ^{-1}\left(R_{\mathrm{tot}} C_{\mathrm{P}} \omega\right)
\end{aligned}
$$


Similarly, for the out of contact case we obtain:

$$
\begin{aligned}
& T_{\mathrm{DC}-\mathrm{o}}=T_{\mathrm{R}}+R_{\mathrm{P}} \dot{q}_{\mathrm{DC}} \\
& T_{\mathrm{AC}-\mathrm{o}}=\frac{R_{\mathrm{P}} \dot{q}_{\mathrm{AC}-\mathrm{o}}}{\sqrt{1+\left(R_{\mathrm{P}} C_{\mathrm{P}} \omega\right)^{2}}} \\
& \varphi_{\mathrm{o}}=-\tan ^{-1}\left(R_{\mathrm{P}} C_{\mathrm{P}} \omega\right)
\end{aligned}
$$

Using Eq. S5 and Eq. S8 the surface temperature can be obtained as:

$$
T_{\mathrm{S}}=\frac{R_{\mathrm{TS}}}{R_{\mathrm{P}}}\left(T_{\mathrm{DC}}-T_{\mathrm{DC}-\mathrm{o}}\right)+T_{\mathrm{DC}}
$$

To use Eq. S11, one can make a single measurement of $T_{\mathrm{DC}-\mathrm{o}}$ before the experiment and assume that it remains constant during scanning. However, in general, $T_{\mathrm{DC}-\mathrm{o}}$ varies with time as the environment temperature drifts (which is sensed as $T_{\mathrm{R}}$ drift), or as the laser power fluctuates (which is sensed as fluctuations in $\left.\dot{q}_{\mathrm{DC}}\right)$. The extent of the aforementioned variations is dependent on the temperature stability of the measurement setup and the intensity stability of the laser source. These variations can be accounted for using Eq. S8, which combined with Eq. S11 leads the following relation.

$$
T_{\mathrm{S}}=\frac{R_{\mathrm{TS}}}{R_{\mathrm{P}}}\left(T_{\mathrm{DC}}-T_{\mathrm{R}}-R_{\mathrm{P}} \dot{q}_{\mathrm{DC}}\right)+T_{\mathrm{DC}}
$$

To obtain $T_{\mathrm{S}}$ using the above equation, the value of $R_{\mathrm{TS}}$ is required. To find $R_{\mathrm{TS}}$, we start by calculating the ratio of probe $\mathrm{AC}$ temperature in out-of-contact to in-contact (during scanning) condition ( $\left.r=T_{\mathrm{AC}-\mathrm{o}} / T_{\mathrm{AC}}\right)$ as shown below. In addition, for generality we include the temperature dependence of probe thermal resistance $\left(R_{\mathrm{P}}\right)$ and lumped thermal capacitance $\left(C_{\mathrm{P}}\right)$. 


$$
r=\frac{T_{\mathrm{AC}-\mathrm{o}}}{T_{\mathrm{AC}}}=\frac{R_{\mathrm{P}}}{R_{\mathrm{tot}}} \sqrt{\frac{1+R_{\mathrm{tot}}^{2} C_{\mathrm{P}}^{2} \omega^{2}}{1+R_{\mathrm{P}}^{2} C_{\mathrm{P}}^{2} \omega^{2}}}
$$

It can be seen that at the limit of fast excitation, $\omega>>\tau_{\mathrm{P}}^{-1}=\left(R_{\mathrm{P}} C_{\mathrm{P}}\right)^{-1}$, the AC temperature signal becomes invariant of thermal contact resistance; therefore, this technique is only useful at low and intermediate heating frequencies (i.e. when $\omega$ is comparable to $\tau_{\mathrm{P}^{-1}}$ ) as shown below:

For slow excitation: $\tau_{P} \omega \ll 1: \frac{T_{A C-o}}{T_{A C}} \approx \frac{R_{P}}{R_{t o t}}$,

For fast excitation: $\tau_{P} \omega \gg 1: \frac{T_{A C-o}}{T_{A C}} \approx 1$.

For intermediate excitation frequencies, the following quadratic equation must be solved to obtain $R_{\mathrm{TS}}$.

$$
R_{\mathrm{TS}}=\frac{R_{\mathrm{P}}}{\sqrt{r^{2}+\left(r^{2}-1\right)\left(R_{\mathrm{P}} C_{\mathrm{P}} \omega\right)^{2}}-1}
$$

After calculation of $R_{\mathrm{TS}}$, the value of $T_{\mathrm{S}}$ can be obtained using Eq. $\mathrm{S} 11$ or $\mathrm{S} 12$, depending on whether the measured values of $T_{\mathrm{R}}$ and $\dot{q}_{\mathrm{DC}}$ are available or not, these quantities are required to use Eq. S12. The quantity $\left(\dot{q}_{\mathrm{DC}}\right)$ has contributions from heat deposited by the integrated probe heater $\left(\dot{q}_{\text {DC-heater }}\right)$ and that deposited by the AFM laser on the probe ( $\left.\dot{q}_{\text {DC-laser }}\right)$ as shown below:

$$
\dot{q}_{\mathrm{DC}}=\dot{q}_{\mathrm{DC}-\text { heater }}+\dot{q}_{\mathrm{DC}-\mathrm{laser}}
$$

In order to account for variations of $\dot{q}_{\mathrm{DC}}$ as a result of fluctuations in laser power, one needs to quantify $\dot{q}_{\text {DC-laser }}$. However, direct measurement of this quantity is nontrivial; therefore, in our measurements, we estimated $\dot{q}_{\text {DC-laser }}$ by measuring the change in temperature of the probe 
when the laser was turned on compared to when it was turned off using the relation: $\dot{q}_{\text {DC-laser }}=\left(T_{\text {DC-laser on }}-T_{\text {DC-laser off }}\right) / R_{\mathrm{P}}$. Subsequently, fluctuations in $\dot{q}_{\text {DC-laser were estimated by }}$ measuring the fluctuations in the total AFM photodetector signal which are proportional to the fluctuations in $\dot{q}_{\text {DC-laser }}$.

\section{S7. Temperature measurement stability and noise}

Figure S5a shows the Allan deviation of the measured sample surface temperature $\left(T_{\mathrm{S}}\right)$ when the probe was parked on a $\mathrm{SiO}_{2}$ substrate (as explained in Figure $3 \mathrm{~d}$ of the manuscript). The black curves demonstrate the measurement stability of $T_{\mathrm{S}}$ without including the effect of fluctuations of deposited laser power (i.e. variations in $\left.\dot{q}_{\mathrm{DC}}\right)$ and reservoir temperature $\left(T_{\mathrm{R}}\right)$ drift. The red curve represents the stability after compensating for the effects of fluctuations in laser power, while the blue curve includes the effects of compensating for both laser power fluctuations and $T_{\mathrm{R}}$ drift. As can be seen, the compensation for laser power fluctuations improves the measurement stability at all timescales (specifically at 5-100 s time scales), whereas compensation for $T_{\mathrm{R}}$ drift improves the stability at timescales $>500 \mathrm{~s}$, achieving a stability of $<10 \mathrm{mK}$ for timescales of $>2 \mathrm{~h}$. Figure S5b shows the amplitude spectrum of temperature corresponding to the same measurement. It can be seen that compensation for laser power also improves the measurement noise at all frequencies specifically around $0.01 \mathrm{~Hz}$. Further, inclusion of $T_{\mathrm{R}}$ compensation very slightly improves the low frequency noise. 

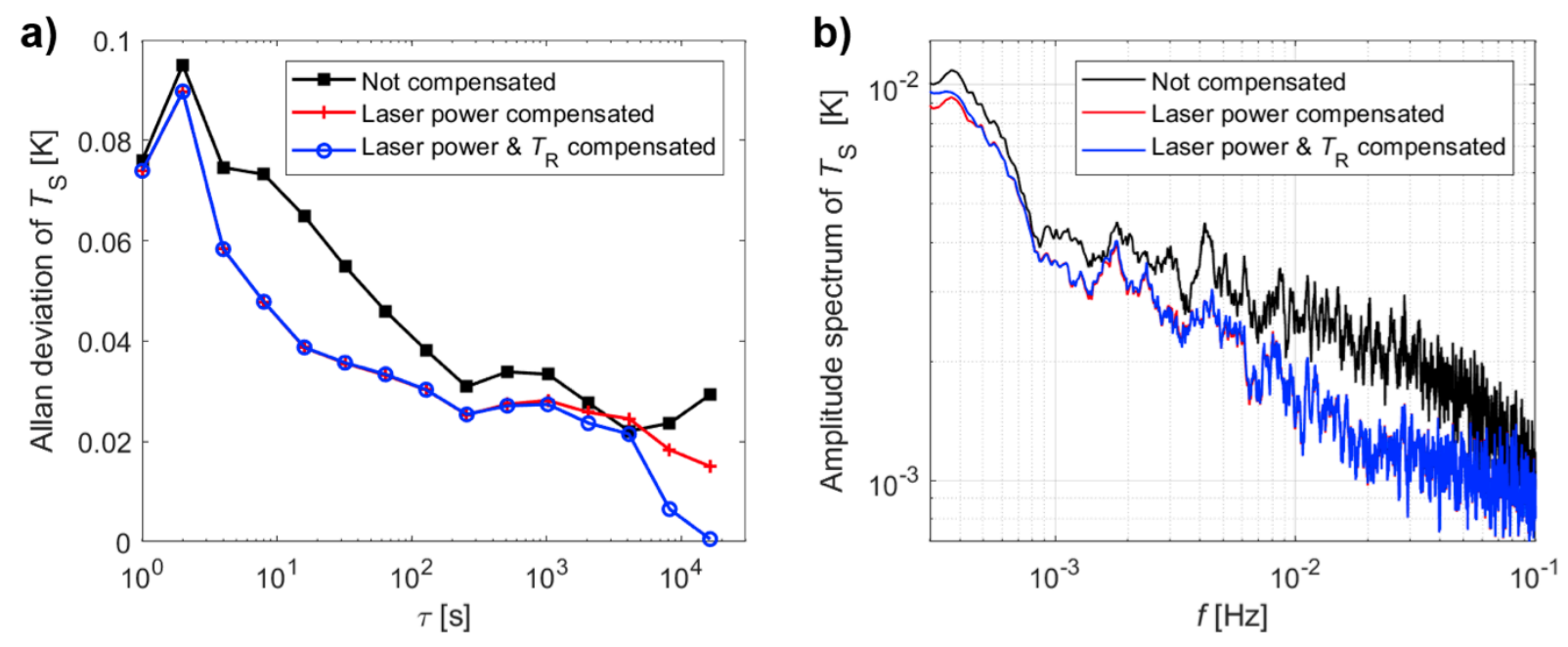

Figure $\mathbf{S 7}$ (a) Allan deviation plot illustrating the stability of the measured sample temperature $\left(T_{S}\right)$ with and without compensation for laser power fluctuations and reservoir temperature $\left(T_{R}\right)$ drift. (b) Amplitude spectrum of measured sample temperature $\left(T_{S}\right)$ for the three cases mentioned above. It is clear from this data that accounting for drift in laser power output and reservoir temperature increases mid to long-term stability and noise.

The observed modest improvement in the long-term drift is due to the low drift of $T_{\mathrm{R}}$ in our vacuum chamber as the experimental room temperature was stabilized to about $0.1 \mathrm{~K}$ (see methods). In a less ideal setup, where there is more drift in the ambient temperature, we expect that the compensation for $T_{\mathrm{R}}$ would have a more beneficial effect at smaller time-scales.

\section{S8. Estimation of spatial resolution of scanning thermal microscopy}

In the manuscript, we estimated the spatial resolution of our SThM scheme using the maximum measurable gradient as observed in Figure 6e, and the temperature sensing resolution via the relation: $\Delta x=\Delta T /(\partial T / \partial x)$ which yields a resolution of $\Delta x \approx 50[\mathrm{mK}] / 31[\mathrm{mK} / \mathrm{nm}]=1.6 \mathrm{~nm}$. In order to check if this estimated resolution can be experimentally realized, we performed a fine line scan perpendicular to the $250 \mathrm{~nm}$-wide Pt heated line sample (the same sample used in the manuscript) with pixel spacing of $0.488 \mathrm{~nm}$ when a DC current of $0.707 \mathrm{~mA}$ was supplied to the Pt line sample. The scanning time was $\sim 500 \mathrm{~ms} /$ pixel similar to the data in the manuscript. To 
extract the spatial resolution, the scanning rate was chosen to be $\sim 1 \mathrm{~nm} / \mathrm{s}$ which was slower than the data in the manuscript (12 nm/s in Figure 4 and 5). The obtained surface temperature profile close to the line edge (a location of large temperature gradient) is shown in Figure S6 (a) with a data point spacing of $0.488 \mathrm{~nm}$. Next, we subsampled the data at a rate of $1 / 3$ using an antialiasing low pass filter to obtain data spacing of $1.46 \mathrm{~nm}$ which is shown in Figure S6 (b). As can be seen, the data in Figure S6 (b) clearly resolves the temperature gradient at the edge of the line with data point spacing of $1.46 \mathrm{~nm}$, which is consistent with the claimed $\sim 2 \mathrm{~nm}$ resolution of this technique. However, for faster scan rates, the estimated spatial resolution would be $6.5 \mathrm{~nm}$ as indicated in the main manuscript. In addition, the spatial resolution is ultimately limited by the tip-sample contact diameter. We estimated the contact diameter using classical Hertzian theory via Eqns. S16 and S17:

$$
\begin{aligned}
& d_{\text {Hertz }}=\left(\frac{6 r P}{E^{*}}\right)^{1 / 3} \\
& E^{*}=\left[\frac{1-v_{1}^{2}}{E_{1}}+\frac{1-v_{2}^{2}}{E_{2}}\right]^{-1}
\end{aligned}
$$

where $r$ is the probe tip radius $(\sim 25 \mathrm{~nm}), P$ is the normal contact force $(\sim 125 \mathrm{nN})$, and $E^{*}$ is the effective elastic modulus obtained from Eq. S17, where $E_{1}=277 \mathrm{GPa}^{7}$ and $E_{2}=165 \mathrm{GPa}^{8}$ are Young's moduli and $v_{1}=0.21^{9}$ and $v_{2}=0.21^{10}$ are the Poisson's ratios of Chromium tip and $\mathrm{Al}_{2} \mathrm{O}_{3}$ coated sample, respectively. For a chromium-coated tip and $\mathrm{Al}_{2} \mathrm{O}_{3}$ surface, the calculated contact diameter is $5.58 \mathrm{~nm}$, which is smaller than the estimated spatial resolution of $\sim 7 \mathrm{~nm}$. Therefore, we estimate the spatial resolution of our technique to be at least $7 \mathrm{~nm}$. 
a)

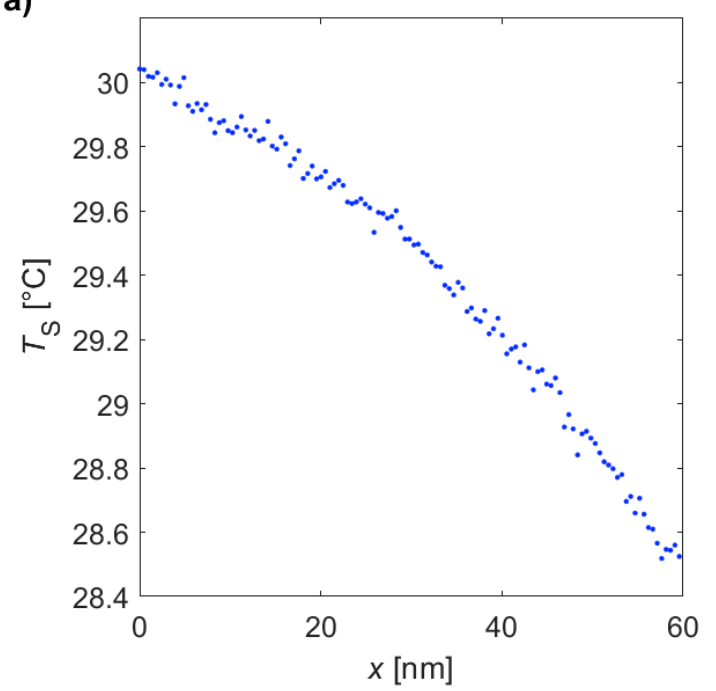

b)

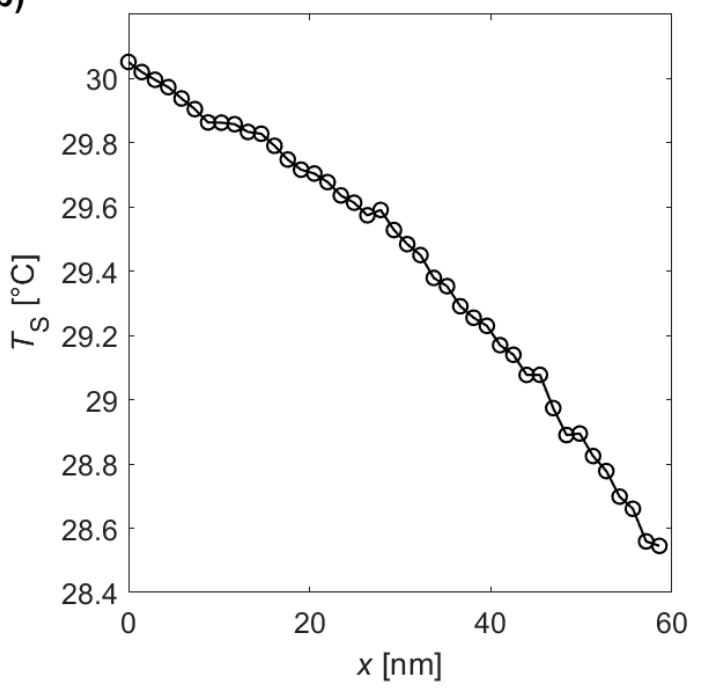

Figure S8 Surface temperature obtained using a line scan perpendicular to a $200 \mathrm{~nm}$-wide Pt line close to the edge of the Pt line (where a significant thermal gradient is present). The surface current corresponds to a situation where an electrical DC current of $0.7 \mathrm{~mA}$ is supplied. (a) Original line scan data with data point spacing of $0.488 \mathrm{~nm}$, (b) Subsampled data at a rate of 1/3, with data point spacing of $1.46 \mathrm{~nm}$. 


\section{S9. Heat transfer model for estimation of temperature profile}

We used a continuum finite element heat transfer model $\left(\mathrm{COMSOL}^{\mathrm{TM}}\right)$ to calculate the temperature profile around the $250 \mathrm{~nm}$-wide self-heated Pt line. A steady-state 2D heat transfer model was used where only half the sample was modeled by taking advantage of symmetric geometry. The computational domain is illustrated in Figure S7 (a), which consists of a structured quadrilateral grid with refinement close to the Pt line where a large thermal gradient is expected. The total length and thickness of the computational domain were $3.5 \mathrm{~mm}$ and $0.5 \mathrm{~mm}$, respectively, which represent the bulk device dimensions. The following thermal conductivity values were used in this model: $148.0 \mathrm{~W} / \mathrm{m} \cdot \mathrm{K}$ for $\mathrm{Si}$ substrate ${ }^{5}, 1.4 \mathrm{~W} / \mathrm{m} \cdot \mathrm{K}$ for $\mathrm{SiO}_{2}$ layer ${ }^{5}$, and $29.5 \mathrm{~W} / \mathrm{m} \cdot \mathrm{K}$ for the thin film Pt layer which is significantly lower than the bulk value. ${ }^{11}$ The boundary conditions set in this model are: (i) a fixed temperature boundary condition $\left(26^{\circ} \mathrm{C}\right)$ at the bottom edge, (ii) symmetric boundary condition (i.e. insulating boundary condition) on the left edges, (iii) insulating boundary condition for all other edges, (iv) volumetric heat source applied to the top Pt layer, and (v) an interfacial thermal resistance of $3.41 \times 10^{-8} \mathrm{~m}^{2} \mathrm{~K} / \mathrm{W}$ was considered between the deposited metal $(\mathrm{Ti} / \mathrm{Pt})$ layer on top and the $\mathrm{SiO}_{2}$ layer based on the experimental results of Ref. ${ }^{12}$. As an example, the obtained temperature profile for a heat source of $6 \times 10^{14} \mathrm{~W} / \mathrm{m}^{3}$ is shown in Figure S7(b) which demonstrates a discontinuity in the temperature field between the top Pt line and the $\mathrm{SiO}_{2}$ layer as a result of interface thermal resistance. Finally, the amplitude of volumetric heat source was adjusted to match the experimentally measured temperature rise of the Pt line in order to obtain the profiles in Figure 6 of the manuscript. 

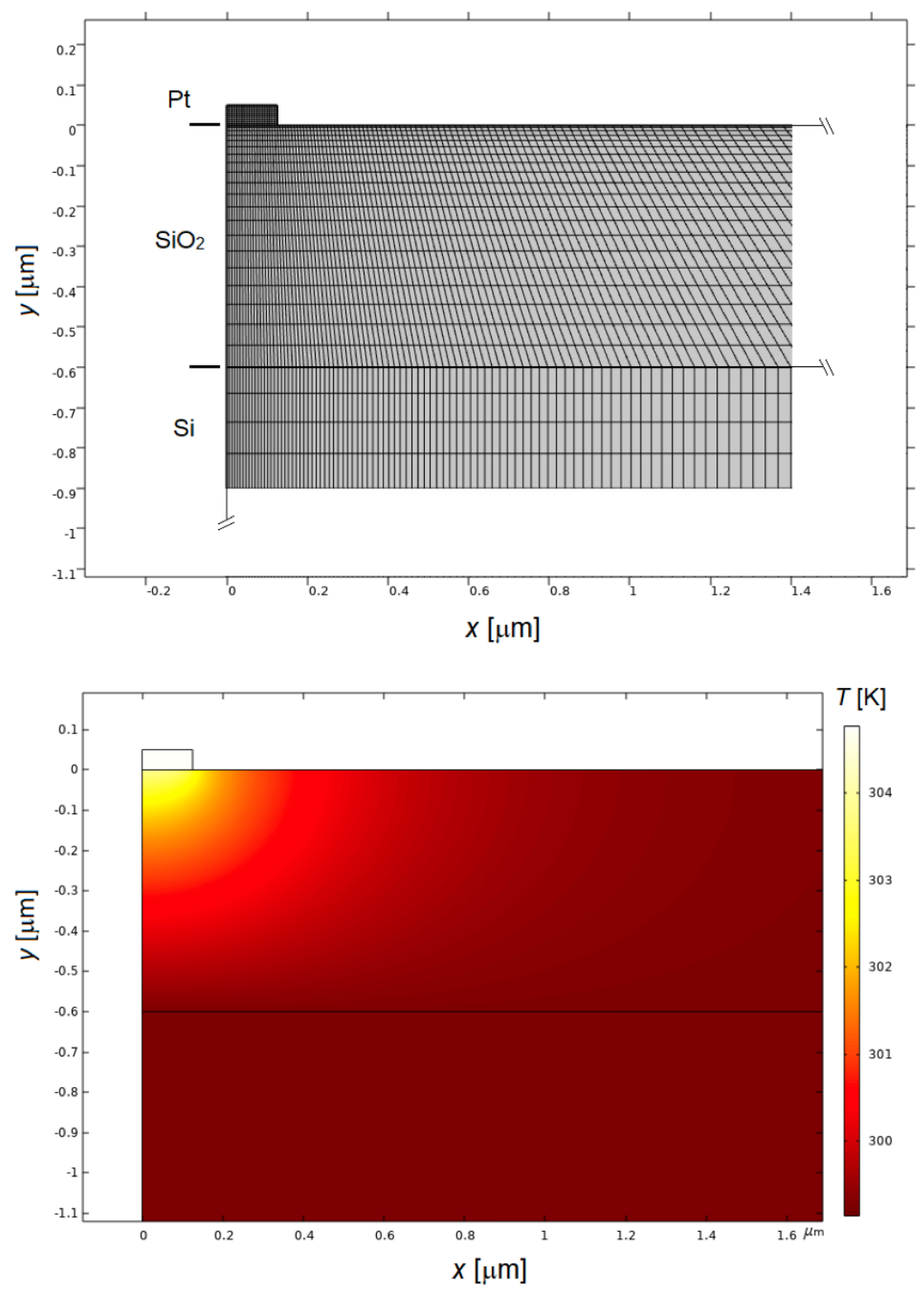

Figure S9 (a) Computational domain for the finite-element heat transfer model. (b) Example of obtained steady-state temperature contour for a volumetric heat source of $6 \times 10^{14} \mathrm{~W} / \mathrm{m}^{3}$ applied on the Pt line.

\section{S10. Histogram analysis of scanning thermal scans}

To analyze the distribution of scanned surface temperature we show in Figure S8a the pixel value histogram obtained from the entire Figure $4 \mathrm{f}$ which shows a standard deviation of $174 \mathrm{mK}$. This variation arises partly from measurement noise and partly due to residual effects from spatial variation of $R_{\mathrm{TS}}$ which although drastically reduced, cannot be completely removed. Similarly, 
Figure $\mathrm{S} 8 \mathrm{~b}$ shows the histogram distribution of pixel values obtained from the dashed box in Figure $5 \mathrm{f}$ which has a standard deviation of $221 \mathrm{mK}$, which is slightly larger than the previous case due to the small temperature gradient generated by heating the Pt line.
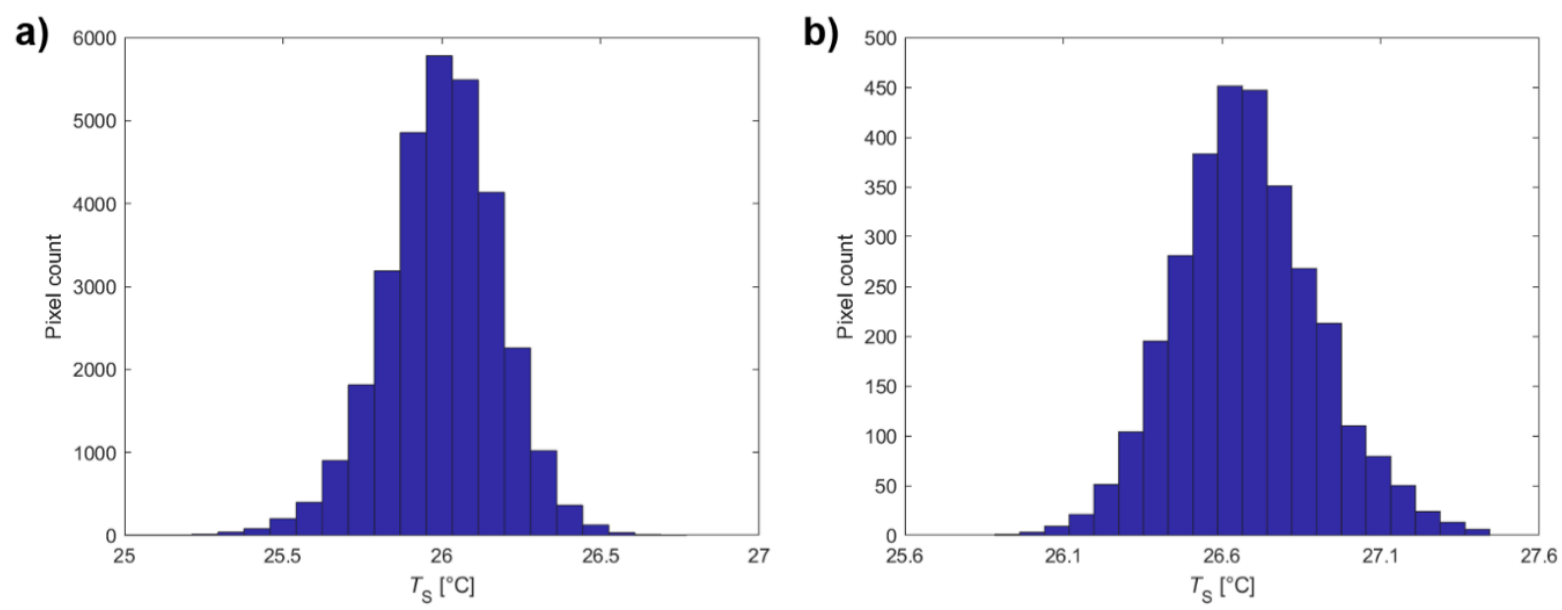

Figure S10 (a) Scanned surface temperature (TS) pixel histogram obtained from: (a) the entire image of Figure4f in the manuscript, which shows a standard deviation of $174 \mathrm{mK}$, and (b) from the pixels inside the dashed box in Figure $5 f$ which shows and standard deviation of $221 \mathrm{mK}$.

\section{S11. Scanning image distortion correction}

As the piezo scanner in our SPM (UHV 750, Beetle STM/AFM, RHK Technology) had a limited lateral ( $x$ and $y$ ) range of $6 \mu \mathrm{m}$, during scanning a large area (comparable to the full range) a distortion in the scanned image was observed as shown in the raw topography scan of Figure S9 (a). This distortion was corrected by applying a sublinear transformation defined as $x_{\text {new }}=\tanh (a$ $\left.x_{\mathrm{old}}\right) / a$ where $a$ is the parameter defining the strength of transformation. A similar transformation was applied to the $y$ direction. Finally, both the $x$ and $y$ coordinates were scaled independently to obtain perpendicular Pt lines (as indicated by SEM images of the device). The distortion-corrected 
topography image is shown in Figure S9 (b). A similar distortion correction procedure was employed to obtain the images of Figure 9 of the manuscript.

a)

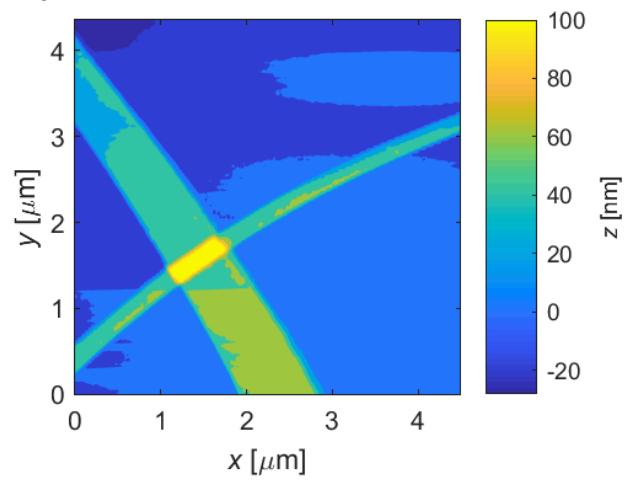

b)

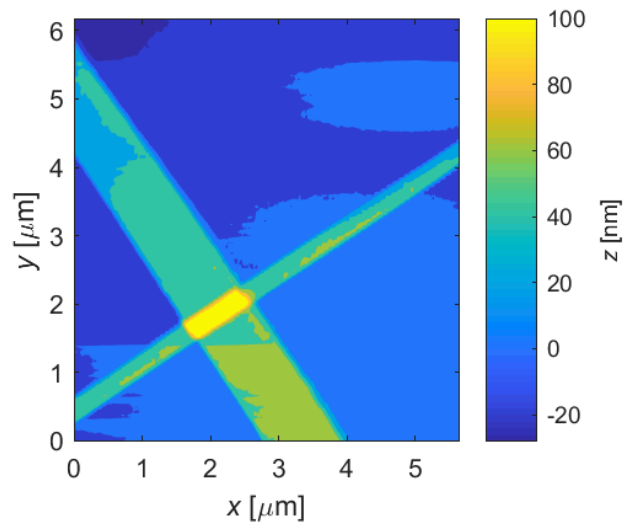

Figure S11 (a) Raw AFM topography image, (b) distortion-corrected topography image.

\section{S12. Temperature profile and characteristic length scales}

The temperature profiles obtained from Figure $7 \mathrm{f}$ along the plotted lines are shown in Figure S10, where the shaded region indicates the standard deviation of averaged pixel values. The characteristic length scale defined by $63 \%$ decay of temperature rise is indicated by red points are $120 \mathrm{~nm}$ and $560 \mathrm{~nm}$ for profile 1 and 2 respectively.

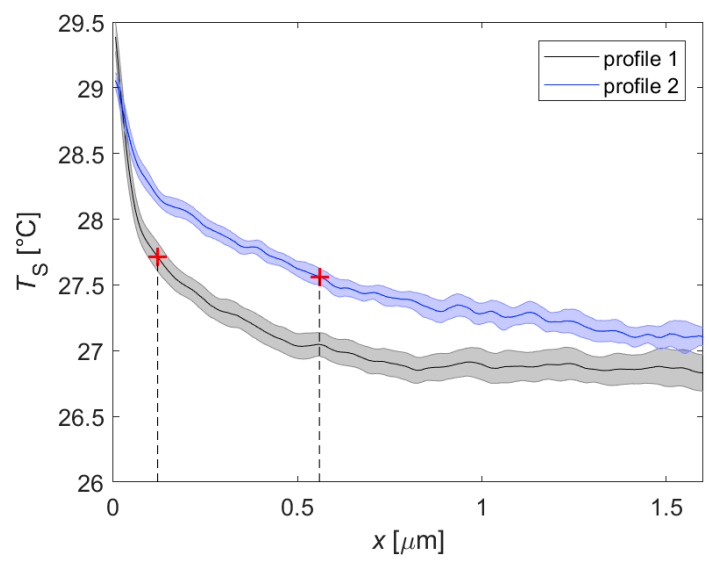

Figure S12 Averaged temperature profiles obtained along the lines plotted in Figure $7 f$. Profile 1 corresponds to temperature distribution on $\mathrm{SiO}_{2}$ substrate, and profile 2 corresponds to the temperature profile on $50 \mathrm{~nm}$ thick Pt line. 


\section{References}

1. Kim, K.; Jeong, W.; Lee, W.; Reddy, P., Ultra-High Vacuum Scanning Thermal Microscopy for Nanometer Resolution Quantitative Thermometry. ACS Nano 2012, 6 (5), 4248-4257.

2. Zhang, X.; Grigoropoulos, C. P., Thermal Conductivity and Diffusivity of Free-Standing Silicon Nitride Thin Films. Rev. Sci. Instrum. 1995, 66 (2), 1115-1120.

3. Ziegler, J. G.; Nichols, N. B., Optimum Settings for Automatic Controllers. Trans. ASME 1942, 64 (11).

4. Sadat, S.; Meyhofer, E.; Reddy, P., High Resolution Resistive Thermometry for Micro/Nanoscale Measurements. Rev. Sci. Instrum. 2012, 83 (8), 084902.

5. DeWitt, D. P.; Bergman, T. L.; Incropera, F. P., Lavine, A. S., Fundamentals of Heat and Mass Transfer, 7th ed.; Wiley: United Kingdom, 2011.

6. Beck, J. V., Large Time Solutions for Temperatures in a Semi-Infinite Body with a Disk Heat Source. Int. J. Heat Mass Transf. 1981, 24 (1), 155-164.

7. Whiting, R.; Angadi, M., Young's Modulus of Thin Films Using a Simplified Vibrating Reed Method. Meas. Sci. Technol. 1990, 1 (7), 662.

8. Purkl, F.; Daus, A.; English, T. S.; Provine, J.; Feyh, A.; Urban, G.; Kenny, T. W., Measurement of Young's Modulus and Residual Stress of Atomic Layer Deposited $\mathrm{Al}_{2} \mathrm{O}_{3}$ and Pt Thin Films. J. Micromech. Microeng. 2017, 27 (8), 085008.

9. Brandes, E. A.; Brook, G., Smithells Metals Reference Book, $7^{\text {th }}$ ed.; Elsevier Science: United Kingdom, 2013.

10. Shackelford, J. F.; Alexander, W., CRC Materials Science and Engineering Handbook, $3^{\text {rd }}$ ed.; CRC Press: United States, 2000.

11. Zhang, X.; Xie, H.; Fujii, M.; Ago, H.; Takahashi, K.; Ikuta, T.; Abe, H.; Shimizu, T., Thermal and Electrical Conductivity of a Suspended Platinum Nanofilm. Appl. Phys. Lett. 2005, 86 (17), 171912.

12. Chien, H.-C.; Yao, D.-J.; Hsu, C.-T. Study for the Interface Thermal Resistance of Metal-Dielectric Layers. In 2008 Second International Conference on Integration and Commercialization of Micro and Nanosystems, Proceedings of the 2008 Second International Conference on Integration and Commercialization of Micro and Nanosystems, Clear Water Bay, Kowloon, Hong Kong, June 3-5, 2008; ASME: New York, 2008; pp 423-427. 